Homology, Homotopy and Applications, vol.14(2), 2012, pp.91-99

\title{
AUTOMORPHISMS OF HURWITZ SERIES
}

\author{
WILLIAM F. KEIGHER AND VARADHARAJ R. SRINIVASAN \\ (communicated by Charles A. Weibel)
}

\begin{abstract}
In this article we will define the notions of Hurwitz automorphism and comorphism of the ring of Hurwitz series. A Hurwitz automorphism is the analog of a Seidenberg automorphism of a power series ring when the characteristic of the underlying ring is not necessarily zero. We will show that the sets of all Hurwitz automorphisms, comorphisms, and derivations of the underlying ring are naturally isomorphic to one another.
\end{abstract}

\section{Introduction}

Let $A$ be a commutative ring with identity and let $H A$ be the ring of Hurwitz series over $A$. In this article, we introduce and study the notions of comorphism and Hurwitz automorphism of $H A$. We show that the set of all derivations on $A$ is naturally isomorphic to both the set of Hurwitz automorphisms of $H A$ (see Theorem 3.6) and the set of comorphisms on $A$ (see Theorem 2.1).

Throughout, all rings are associative, commutative and unitary, and $A$ and $B$ will typically denote rings. If $f: X \rightarrow Y$ is a function, then we will occasionally use the notation $f: X \rightarrow Y: x \mapsto f(x)$ to describe the action of $f$ on elements $x \in X$. The natural numbers $\{0,1,2, \ldots\}$ will be denoted by $\mathbb{N}$, and similarly $\mathbb{Q}$ and $\mathbb{C}$ will denote the rational numbers and complex numbers, respectively. For any $m, n \in \mathbb{N}, \delta_{n}^{m}$ will denote the Kronecker delta, i.e., $\delta_{n}^{m}=1$ if $m=n$ and $\delta_{n}^{m}=0$ if $m \neq n$.

\section{Definitions and Conventions}

If $A$ is a ring, then a derivation on $A$ is an additive mapping $d: A \rightarrow A$ such that, for all $a, b \in A, d(a b)=d(a) b+a d(b)$. Examples include the familiar $d / d t$ on the ring $\mathbb{C}[t]$ of polynomials in $t$ with coefficients in $\mathbb{C}$, and for any ring $A$, the trivial derivation $0_{A}$ defined by $0_{A}(a)=0$ for any $a \in A$. The set of all derivations of $A$ will be denoted by Der $A$. A differential ring consists of a pair $(A, d)$, where $A$ is a ring and $d$ is a derivation on $A$. If $\left(A, d_{1}\right)$ and $\left(B, d_{2}\right)$ are differential rings, then a differential ring homomorphism $f:\left(A, d_{1}\right) \rightarrow\left(B, d_{2}\right)$ is a ring homomorphism $f: A \rightarrow B$ such that $d_{2} \circ f=f \circ d_{1}$.

The following result is probably well-known, but we record it here, as it will be useful later. The proof is immediate.

Received May 4, 2012; published on November 8, 2012.

2000 Mathematics Subject Classification: 12H05, $12 \mathrm{H} 20$.

Key words and phrases: automorphism, comorphism, derivation, Hurwitz series.

Article available at http://intlpress.com/HHA/v14/n2/a6 and doi:10.4310/HHA.2012.v14.n2.a6

Copyright (C) 2012, International Press. Permission to copy for private use granted. 
Lemma 1.1. Suppose that $(A, d)$ is a differential ring and $f: A \rightarrow B$ and $g: B \rightarrow A$ are ring homomorphisms such that $f \circ g=\operatorname{id}_{B}$. Then $f \circ d \circ g$ is a derivation on $B$.

\section{Ring of Hurwitz series}

From [1] we recall that for any ring $A$, the ring of Hurwitz series over $A$, denoted by $H A$, consists of sequences $h=\left(h_{0}, h_{1}, h_{2}, \ldots\right)$, where $h_{n} \in A$ for each $n \in \mathbb{N}$. It is often convenient to view a Hurwitz series as a function $h: \mathbb{N} \rightarrow A: n \mapsto h(n)$. Let $g, h \in H A$. Addition in $H A$ is defined termwise, i.e.,

$$
(g+h)(n)=g(n)+h(n),
$$

and the Hurwitz product of $g$ and $h$ is given by

$$
(g \cdot h)(n)=\sum_{k=0}^{n}\left(\begin{array}{l}
n \\
k
\end{array}\right) g(k) h(n-k)
$$

for all $n \in \mathbb{N}$, where $\left(\begin{array}{l}n \\ k\end{array}\right)$ denotes the binomial coefficient.

The ring $H A$ is a differential ring with derivation

$$
\partial_{A}: H A \rightarrow H A:\left(h_{0}, h_{1}, h_{2}, \ldots\right) \mapsto\left(h_{1}, h_{2}, h_{3}, \ldots\right),
$$

that is, $\partial_{A}$ is the left shift operator. Observe that if $d$ is a derivation on $A$, then

$$
H d: H A \rightarrow H A:\left(h_{0}, h_{1}, h_{2}, \ldots\right) \mapsto\left(d\left(h_{0}\right), d\left(h_{1}\right), d\left(h_{2}\right), \ldots\right)
$$

is a derivation on $H A$, and $H d \circ \partial=\partial \circ H d$. For any ring $A$, there are natural ring homomorphisms

$$
\lambda_{A}: A \rightarrow H A: a \mapsto(a, 0,0, \ldots)
$$

and

$$
\varepsilon_{A}: H A \rightarrow A:\left(h_{0}, h_{1}, h_{2}, \ldots\right) \mapsto h_{0} .
$$

Furthermore, if $d$ is a derivation on $A$ then

$$
\widetilde{d}: A \rightarrow H A: a \mapsto\left(a, d(a), d^{2}(a), \ldots\right)
$$

is also a ring homomorphism, called the Hurwitz homomorphism of $d$. Note that $\widetilde{0}_{A}=\lambda_{A}$. If $f: A \rightarrow B$ is a ring homomorphism, then $H f: H A \rightarrow H B$ is defined as follows: for $h=\left(h_{0}, h_{1}, h_{2}, \ldots\right), H f(h)=\left(f\left(h_{0}\right), f\left(h_{1}\right), f\left(h_{2}\right), \ldots\right)$.

For convenience and when there is no ambiguity, we will often use $\varepsilon, \lambda$ and $\partial$ instead of $\varepsilon_{A}, \lambda_{A}$ and $\partial_{A}$ respectively.

\section{Divided powers}

From $[\mathbf{2}]$ we recall that for any ring $A$, the divided powers $x^{[i]}$ in $H A$, for $i \in \mathbb{N}$, are defined by

$$
x^{[i]}(n):=\delta_{n}^{i},
$$

so that $x^{[0]}=1_{H A}, x^{[1]}=(0,1,0, \ldots), x^{[2]}=(0,0,1,0, \ldots)$, etc. The following results are easy to check:

$$
x^{[m]} \cdot x^{[n]}=\left(\begin{array}{c}
m+n \\
n
\end{array}\right) x^{[m+n]}, \quad \forall m, n \in \mathbb{N}
$$


and

$$
\text { for any } h \in H A,\left(h \cdot x^{[k]}\right)(n)= \begin{cases}0, & \text { if } n<k ; \\
\left(\begin{array}{l}
n \\
k
\end{array}\right) h(n-k), & \text { otherwise. }\end{cases}
$$

We define the order of $0 \neq h \in H A$, denoted by ord $(h)$, to be the minimum $i \in \mathbb{N}$ such that $h(i) \neq 0$ and when $h=0, \operatorname{ord}(h):=\infty$. Using this order, one can define a metric $\delta$ on $H A$ by $\delta(g, h)=\left(\frac{1}{2}\right)^{\operatorname{ord}(g-h)}$; see [2]. Using this topology on $H A$ and the divided powers $x^{[i]}$, it is easy to see that for any $h \in H A, h=\sum_{n=0}^{\infty} h(n) x^{[n]}$.

\section{Comor $A$ and Haut $A$}

A comorphism $\alpha$ on a ring $A$ is a ring homomorphism $\alpha: A \rightarrow H A$ such that the diagrams

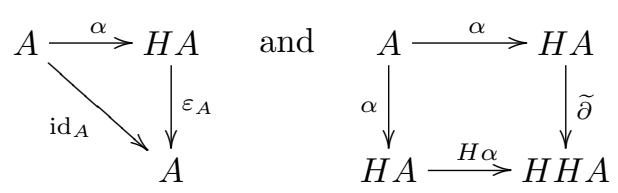

commute. Examples of comorphisms on $A$ include $\lambda_{A}$ and $\widetilde{d}$, where $d$ is a derivation on $A$. The set of all comorphisms on $A$ will be denoted by Comor $A$.

It is well-known that if $A$ is a differential ring with derivation $d$ and $\mathbb{Q} \subseteq A$, then there is a differential ring homomorphism

$$
T:(A, d) \rightarrow(A[[t]], d / d t): a \mapsto \sum_{n=0}^{\infty} \frac{d^{(n)}(a)}{n !} t^{n}
$$

called the Taylor homomorphism of $d$. From the ring homomorphism

$$
A[[t]] \rightarrow A: \sum_{n=0}^{\infty} a_{n} t^{n} \mapsto a_{0},
$$

by Proposition 2.1 of [1] we get a natural differential ring homomorphism

$$
\psi_{A}:(A[[t]], d / d t) \rightarrow\left(H A, \partial_{A}\right): \sum_{n=0}^{\infty} a_{n} t^{n} \mapsto\left(n ! a_{n}\right) .
$$

When $\mathbb{Q} \subset A$, the Taylor homomorphism $T$ and the Hurwitz homomorphism $\widetilde{d}$ are related by $\psi_{A}$ via the commutative diagram

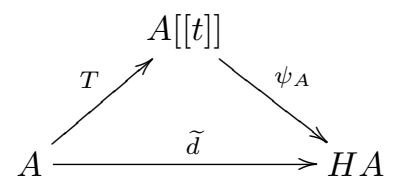

and, moreover, $\psi_{A}$ is an isomorphism. However, the Taylor homomorphism $T$ is defined only in case $\mathbb{Q} \subseteq A$, while the Hurwitz homomorphism $\widetilde{d}$ is defined for any differential ring $A$ of any characteristic. 
A ring endomorphism $\sigma$ of $H A$ is called a Hurwitz endomorphism if, for all $n \in \mathbb{N}$, $\sigma$ satisfies the following conditions:

$$
\begin{aligned}
(\varepsilon \circ \partial \circ \sigma \circ \lambda)^{n} & =\varepsilon \circ \partial^{n} \circ \sigma \circ \lambda, \\
\sigma\left(x^{[n]}\right) & =x^{[n]}, \\
\operatorname{ord}(h) & \leqslant \operatorname{ord}(\sigma(h)) .
\end{aligned}
$$

We note that the condition (3) is equivalent to $\sigma \circ \lambda \in$ Comor $A$ and that the condition (5) guarantees the continuity of $\sigma$ with respect to the metric $\delta$. Furthermore, if $\sigma$ is bijective then we call $\sigma$ a Hurwitz automorphism of $H A$. The set of all Hurwitz automorphisms of $H A$ will be denoted by Haut $A$.

The next two sections of this article are dedicated to proving the equivalence between Der $A$, Comor $A$ and Haut $A$.

\section{Equivalence of $\operatorname{Der} A$ and Comor $A$}

The following theorem shows that Der $A$ and Comor $A$ are equivalent as sets. From the definition of a comorphism and from Lemma 1.1, we see that $\varepsilon_{A} \circ \partial_{A} \circ \alpha$ is a derivation on $A$ for any $\alpha \in$ Comor $A$.

Theorem 2.1. Consider the mappings $\Omega$ : Der $A \rightarrow$ Comor $A$ defined by $\Omega(d)=\widetilde{d}$ and $\Delta$ : Comor $A \rightarrow \operatorname{Der} A$ defined by $\Delta(\alpha)=\varepsilon \circ \partial \circ \alpha$. Then $\Delta \circ \Omega=\operatorname{id}_{\operatorname{Der} A}$ and

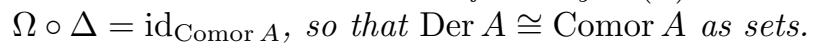

Proof. It is easy to see that $\varepsilon(\partial(\widetilde{d}(a)))=d(a)$ for any $a \in A$. Thus $\Delta(\Omega(d))=d$.

For $\alpha \in \operatorname{Comor} A$ and $a \in A$, we have

$$
\Omega(\Delta(\alpha))(a)=\left(a, \varepsilon \circ \partial \circ \alpha(a),(\varepsilon \circ \partial \circ \alpha)^{2}(a), \ldots\right) .
$$

From the definition of Comor $A$, we have $\widetilde{\partial} \circ \alpha(a)=H \alpha \circ \alpha(a)$, which in turn gives us the relation, for any $n \in \mathbb{N}$,

$$
\partial^{n} \circ \alpha=\alpha \circ \varepsilon \circ \partial^{n} \circ \alpha .
$$

A straightforward computation, using the above equation, will give us the relation $\varepsilon \circ$ $\partial^{n} \circ \alpha=(\varepsilon \circ \partial \circ \alpha)^{n}$. Note that $\varepsilon \circ \partial^{n} \circ \alpha(a)=\alpha(a)(n)$. Thus it follows that $\alpha(a)=$ $\Omega(\varepsilon \circ \partial \circ \alpha)(a)$, and thus $\Omega(\Delta(\alpha))=\alpha$.

\section{Equivalence of Der $A$ and Haut $A$}

In this section, we will show that there is a natural isomorphism between the sets Der $A$ and Haut $A$.

Lemma 3.1. Let $\sigma \in$ Haut $A, a \in A, k \in \mathbb{N}, h \in H A$ and define $d_{\sigma}$ by $d_{\sigma}:=\varepsilon \circ \partial \circ$ $\sigma \circ \lambda$. Then

$$
\text { 1. } \sigma(\lambda(a))=\sigma\left(a x^{[0]}\right)=\left(a, d_{\sigma}(a), d_{\sigma}^{2}(a), \ldots\right),
$$


2. $d_{\sigma}$ is a derivation on $A$ and $\sigma(\lambda(a))=\widetilde{d}_{\sigma}(a)$. That is, the diagram

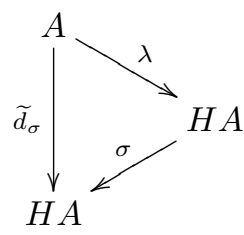

commutes, and

3. $\sigma\left(a x^{[k]}\right)(n)= \begin{cases}0, & \text { if } n<k ; \\ \left(\begin{array}{l}n \\ k\end{array}\right) d_{\sigma}^{n-k}(a), & \text { if } n \geqslant k .\end{cases}$

Proof. Item 1. follows immediately from equation (3). From item 1. it follows that $\varepsilon \circ \sigma \circ \lambda=\operatorname{id}_{A}$. Thus from Lemma 1.1 we obtain that $d_{\sigma}$ is a derivation on $A$. Since $\sigma$ is a homomorphism, we have $\sigma\left(a x^{[k]}\right)=\sigma\left(a x^{[0]}\right) \cdot \sigma\left(x^{[k]}\right)$, and since $\sigma\left(x^{[k]}\right)=x^{[k]}$, we have

$$
\sigma\left(a x^{[k]}\right)=\sigma\left(a x^{[0]}\right) \cdot x^{[k]} .
$$

Now from item 1 . of this lemma and from equation (1), item 3. follows.

Theorem 3.2. Let $\sigma \in \operatorname{Haut} A$ and $h \in H A$. Then for each $n \in \mathbb{N}$,

$$
\sigma(h)(n)=\sum_{k=0}^{n}\left(\begin{array}{l}
n \\
k
\end{array}\right) d_{\sigma}^{n-k}(h(k)),
$$

where $d_{\sigma}$ is the derivation given by $d_{\sigma}:=\varepsilon \circ \partial \circ \sigma \circ \lambda$.

Proof. Let $h \in H A$ and write $h=\sum_{k=0}^{\infty} h(k) x^{[k]}$. Then

$$
\begin{aligned}
\sigma(h)(n) & =\sigma\left(\sum_{k=0}^{n} h(k) a^{[k]}\right)(n)+\sigma\left(\sum_{k=n+1}^{\infty} h(k) x^{[k]}\right)(n) \\
& =\sum_{k=0}^{n} \sigma\left(h(k) x^{[k]}\right)(n)+\sigma\left(\sum_{k=n+1}^{\infty} h(k) x^{[k]}\right)(n) .
\end{aligned}
$$

Now since the ord $\left(\sum_{k=n+1}^{\infty}(h(k)) x^{[k]}\right) \geqslant n+1$, from condition (5), we obtain that $\sigma\left(\sum_{k=n+1}^{\infty}(h(k)) x^{[k]}\right)(t)=0$ for all $t \leqslant n$. Now from Lemma 3.1 item 3., it follows that $\sigma(h)(n)=\sum_{k=0}^{n}\left(\begin{array}{l}n \\ k\end{array}\right) d_{\sigma}^{n-k}(h(k))$.

For any $d \in \operatorname{Der} A, h \in H A$, and $n \in \mathbb{N}$, define

$$
\sigma_{d}(h)(n)=\sum_{k=0}^{n}\left(\begin{array}{l}
n \\
k
\end{array}\right) d^{n-k}(h(k)) .
$$

In the next few results, we will show that $\sigma_{d}$ is a Hurwitz automorphism.

Theorem 3.3. For any $d \in \operatorname{Der} A, \sigma_{d}$ is a Hurwitz endomorphism of $H A$. 
Proof. From the fact that $d^{n}$ is additive, it is easy to see that $\sigma_{d}$ is also additive. Let $h=\left(h_{0}, h_{1}, \ldots\right), g=\left(g_{0}, g_{1}, \ldots\right) \in H A$ and from equation (7), we have

$$
\begin{aligned}
\left(\sigma_{d}(h) \cdot \sigma_{d}(g)\right)(n) & =\sum_{k=0}^{n}\left(\begin{array}{l}
n \\
k
\end{array}\right) \sigma_{d}(h)(k) \sigma_{d}(g)(n-k) \\
& =\sum_{k=0}^{n}\left(\begin{array}{l}
n \\
k
\end{array}\right)\left(\sum_{j=0}^{k}\left(\begin{array}{l}
k \\
j
\end{array}\right) d^{k-j}\left(h_{j}\right)\right)\left(\sum_{i=0}^{n-k}\left(\begin{array}{c}
n-k \\
i
\end{array}\right) d^{n-k-i}\left(g_{i}\right)\right) \\
& =\sum_{k=0}^{n} \sum_{j=0}^{k} \sum_{i=0}^{n-k}\left(\begin{array}{l}
n \\
k
\end{array}\right)\left(\begin{array}{c}
k \\
j
\end{array}\right)\left(\begin{array}{c}
n-k \\
i
\end{array}\right) d^{k-j}\left(h_{j}\right) d^{n-k-i}\left(g_{i}\right) .
\end{aligned}
$$

On the other hand, since $(h \cdot g)(p)=\sum_{q=0}^{p}\left(\begin{array}{c}p \\ q\end{array}\right) h_{q} g_{p-q}$, we have

$$
\begin{aligned}
\sigma(h \cdot g)(n) & =\sum_{p=0}^{n}\left(\begin{array}{l}
n \\
p
\end{array}\right) d^{n-p}\left(\sum_{q=0}^{p}\left(\begin{array}{l}
p \\
q
\end{array}\right) h_{q} g_{p-q}\right) \\
& =\sum_{p=0}^{n} \sum_{q=0}^{p} \sum_{r=0}^{n-p}\left(\begin{array}{c}
n \\
p
\end{array}\right)\left(\begin{array}{c}
p \\
q
\end{array}\right)\left(\begin{array}{c}
n-p \\
r
\end{array}\right) d^{n-p-r}\left(h_{q}\right) d^{r}\left(g_{p-q}\right) .
\end{aligned}
$$

We will now show that equations (8) and (9) are identical. Note that both the equations (8) and (9) have the same number of terms. Consider the equations $n-p-r=$ $k-j, r=n-k-i, q=j$ and $p-q=i$. Solving for $p, q$ and $r$, we obtain $q=j$, $p=i+j, r=n-k-i$. Substituting for $p, q$ and $r$ in $\left(\begin{array}{l}n \\ p\end{array}\right)\left(\begin{array}{l}p \\ q\end{array}\right)\left(\begin{array}{c}n-p \\ r\end{array}\right)$, we obtain

$$
\left(\begin{array}{c}
n \\
i+j
\end{array}\right)\left(\begin{array}{c}
i+j \\
j
\end{array}\right)\left(\begin{array}{c}
n-i-j \\
n-k-i
\end{array}\right)=\frac{n !}{i ! j !(k-j) !(n-k-i) !} .
$$

On the other hand,

$$
\left(\begin{array}{l}
n \\
k
\end{array}\right)\left(\begin{array}{c}
k \\
j
\end{array}\right)\left(\begin{array}{c}
n-k \\
i
\end{array}\right)=\frac{n !}{i ! j !(k-j) !(n-k-i) !} .
$$

Thus $\sigma_{d}$ is a ring endomorphism. From the definition of $\sigma_{d}$, it is clear that $\sigma_{d}\left(x^{[k]}\right)=$ $x^{[k]}$ and $\sigma_{d}\left(a x^{[0]}\right)(n)=d^{n}(a)$, and thus $\sigma_{d}\left(a x^{[0]}\right)=\left(a, d(a), d^{2}(a), \ldots\right)$. Since $\lambda(a)=$ $a x^{[0]}$, it is now easy to check that $\varepsilon \circ \partial \circ \sigma_{d} \circ \lambda=d$ and that $\varepsilon \circ \partial^{n} \circ \sigma_{d} \circ \lambda=d^{n}$ for any $n$. Hence $\sigma_{d}$ is a Hurwitz endomorphism.

Lemma 3.4. If $d_{1}, d_{2} \in \operatorname{Der} A$ with $d_{1} \circ d_{2}=d_{2} \circ d_{1}$, then $\sigma_{d_{1}} \circ \sigma_{d_{2}}=\sigma_{d_{1}+d_{2}}=\sigma_{d_{2}}$ $\circ \sigma_{d_{1}}$.

Proof. Let $h=\left(h_{0}, h_{1}, \ldots\right) \in H A$. Then

$$
\begin{aligned}
\sigma_{d_{2}}\left(\sigma_{d_{1}}(h)\right)(n) & =\sum_{k=0}^{n}\left(\begin{array}{l}
n \\
k
\end{array}\right) d_{2}^{n-k}\left(\sum_{i=0}^{k}\left(\begin{array}{l}
k \\
i
\end{array}\right) d_{1}^{k-i}\left(h_{i}\right)\right) \\
& =\sum_{k=0}^{n} \sum_{i=0}^{k}\left(\begin{array}{l}
n \\
k
\end{array}\right)\left(\begin{array}{l}
k \\
i
\end{array}\right) d_{2}^{n-k}\left(d_{1}^{k-i}\left(h_{i}\right)\right) .
\end{aligned}
$$


Similarly,

$$
\begin{aligned}
\sigma_{d_{1}+d_{2}}(h)(n) & =\sum_{j=0}^{n}\left(\begin{array}{l}
n \\
j
\end{array}\right)\left(d_{1}+d_{2}\right)^{n-j}\left(h_{j}\right) \\
& =\sum_{j=0}^{n}\left(\begin{array}{c}
n \\
j
\end{array}\right)\left(\sum_{l=0}^{n-j}\left(\begin{array}{c}
n-j \\
l
\end{array}\right) d_{1}^{n-j-l}\left(d_{2}^{l}\left(h_{j}\right)\right)\right) \\
& =\sum_{j=0}^{n} \sum_{l=0}^{n-j}\left(\begin{array}{c}
n \\
j
\end{array}\right)\left(\begin{array}{c}
n-j \\
l
\end{array}\right) d_{1}^{n-j-l}\left(d_{2}^{l}\left(h_{j}\right)\right) .
\end{aligned}
$$

Note that when $j=i$ and $l=n-k$, we have $d_{1}^{n-j-l}\left(d_{2}^{l}\left(h_{j}\right)\right)=d_{2}^{n-k}\left(d_{1}^{k-i}\left(h_{i}\right)\right)$. Now substituting $j=i$ and $l=n-k$ in $\left(\begin{array}{c}n \\ j\end{array}\right)\left(\begin{array}{c}n-j \\ l\end{array}\right)$, we obtain $\frac{n !}{i !(k-i) !(n-k) !}$. On the other hand, $\left(\begin{array}{l}n \\ k\end{array}\right)\left(\begin{array}{c}k \\ i\end{array}\right)=\frac{n !}{i !(k-i) !(n-k) !}$. Thus $\sigma_{d_{1}+d_{2}}=\sigma_{d_{2}} \circ \sigma_{d_{1}}$. Similarly, it follows that $\sigma_{d_{1}+d_{2}}$ $=\sigma_{d_{1}} \circ \sigma_{d_{2}}$.

Theorem 3.5. For any $d \in \operatorname{Der} A, \sigma_{d}$ is a Hurwitz automorphism of $H A$ and $\sigma_{d}^{-1}=$ $\sigma_{-d}$.

Proof. We only need to show that $\sigma_{d}$ has an inverse for each $d$. It is easy to check that the automorphism corresponding to the trivial derivation, $0_{A}$, is the identity map $\operatorname{id}_{H A}$. Since $d$ and $-d$ are commuting derivations, it follows from Lemma 3.4 that $\operatorname{id}_{H A}=\sigma_{0_{A}}=\sigma_{d+(-d)}=\sigma_{d} \circ \sigma_{-d}$. Thus $\sigma_{d}$ is a Hurwitz automorphism with inverse $\sigma_{-d}$.

Theorem 3.6. Let $\Phi: \operatorname{Der} A \rightarrow$ Haut $A$ and $\Psi:$ Haut $A \rightarrow \operatorname{Der} A$ be defined by $\Phi(d)$ $=\sigma_{d}$ and $\Psi(\sigma)=d_{\sigma}$, where $d_{\sigma}:=\varepsilon \circ \partial \circ \sigma \circ \lambda$. Then $\Phi \circ \Psi=\operatorname{id}_{\text {Haut } A}$ and $\Psi \circ \Phi=$ $\operatorname{id}_{\text {Der } A}$. Thus Der $A$ and Haut $A$ are isomorphic sets.

Proof. For any $\sigma \in$ Haut $A, \Phi(\Psi(\sigma))=\Phi\left(d_{\sigma}\right)=\sigma_{d_{\sigma}}$. Note that for $h=\left(h_{0}, h_{1}, \ldots\right)$, $\sigma_{d_{\sigma}}(h)(n)=\sum_{k=0}^{n}\left(\begin{array}{l}n \\ k\end{array}\right) d_{\sigma}^{n-k}\left(h_{k}\right)$. But from the definition of $\sigma$, we know that $\sigma(h)(n)=$ $\sum_{k=0}^{n}\left(\begin{array}{l}n \\ k\end{array}\right) d_{\sigma}^{n-k}\left(h_{k}\right)$. Thus $\Phi(\Psi(\sigma))=\sigma$.

For $d \in \operatorname{Der} A, \Psi(\Phi(d))=\Psi\left(\sigma_{d}\right)=d_{\sigma_{d}}$ and for any $a \in A$, we know that $\sigma_{d}(\lambda(a))$ $=\left(a, d(a), d^{2}(a), \ldots\right)$ and thus $d_{\sigma_{d}}(a)=\varepsilon \circ \partial \circ \sigma_{d} \circ \lambda(a)=d(a)$. Thus $\Psi(\Phi(d))=d$. Since $\Psi$ is the inverse of $\Phi$, it follows that $\Phi$ and $\Psi$ are isomorphisms (of sets).

\section{Commuting derivations and Hurwitz automorphisms}

We first recall that a ring $A$ is said to have no 2 -torsion if for any $a \in A$, if $2 a=0$, then $a=0$. It is clear that such a ring $A$ is not of characteristic 2 , and that if 2 is invertible in $A$, then $A$ has no 2-torsion.

In this section we will show that if $\Delta \subset \operatorname{Der} A$ is a subgroup of Der $A$ consisting of commuting derivations, then $\Phi(\Delta) \subset$ Haut $A$ is an abelian group (with respect to $\circ$ ). We will also show that if $G \subset$ Haut $A$ is an abelian group and if $A$ has no 2-torsion, then $\Delta=\left\{d_{\sigma} \mid \sigma \in G\right\}$ is a subgroup of Der $A$ consisting of commuting derivations. 
Theorem 4.1. Let $\Delta \subset$ Der $A$ be a subgroup of Der $A$ consisting of commuting derivations. Then $\Phi(\Delta) \subset$ Haut $A$ is an abelian group (with respect to $\circ$ ). Moreover,

$$
\left.\Phi\right|_{\Delta}:(\Delta,+) \rightarrow(\Phi(\Delta), \circ)
$$

is a group isomorphism.

Proof. Let $\sigma_{1}, \sigma_{2} \in \Phi(\Delta)$. For notational convenience, let $d_{i}:=d_{\sigma_{i}}$ for $i=1,2$. Then from Theorem 3.6, we know that $d_{1}, d_{2} \in \Delta$ and that $\sigma_{d_{i}}=\sigma_{i}$ for $i=1,2$. Now applying Lemma 3.4, we obtain that

$$
\Phi\left(d_{1}+d_{2}\right)=\sigma_{d_{1}+d_{2}}=\sigma_{d_{1}} \circ \sigma_{d_{2}}=\sigma_{1} \circ \sigma_{2}=\Phi\left(d_{1}\right) \circ \Phi\left(d_{2}\right) .
$$

Now since $d_{1}+d_{2} \in \Delta$, we have $\sigma_{1} \circ \sigma_{2} \in \Phi(\Delta)$.

Let $\sigma \in \Phi(\Delta)$. From Theorem 3.6, we know that $d_{\sigma} \in \Delta$ and $\sigma=\sigma_{d_{\sigma}}$. But from Theorem 3.5 we know that $\sigma_{-d_{\sigma}}$ is the inverse of $\sigma$. Since $-d_{\sigma} \in \Delta$, we have $\sigma_{-d_{\sigma}} \in$ $\Phi(\Delta)$. Hence $\Phi(\Delta)$ forms a group. Now from Lemma 3.4, it follows that $\Phi(\Delta)$ is an abelian group. Also note that for any $d \in \operatorname{Der} A, \Phi\left(0_{A}\right)=\sigma_{0_{A}}=\sigma_{d+-d}=\sigma_{d} \circ \sigma_{-d}=$ $\operatorname{id}_{H A}$. Thus $\left.\Phi\right|_{\Delta}$ is a group isomorphism.

Theorem 4.2. Let $A$ be a ring having no 2-torsion and let $G \subset$ Haut $A$ be an abelian group. Then $\Psi(G)=\left\{d_{\sigma} \mid \sigma \in G\right\}$ is a subgroup of Der $A$ consisting of commuting derivations. Moreover, $\left.\Psi\right|_{G}:(G, \circ) \rightarrow(\Psi(G),+)$ is a group isomorphism.

Proof. Let $\sigma_{1}, \sigma_{2} \in G$ and let $a \in A$. For notational convenience, let $d_{i}:=d_{\sigma_{i}}$ for $i=1,2$. Since $\sigma_{1}$ and $\sigma_{2}$ commute, we have $\left.\sigma_{1}\left(\sigma_{2}(\lambda(a))\right)(n)=\sigma_{2}\left(\sigma_{1}(\lambda(a))\right)\right)(n)$ for all $n$. That is,

$$
\sum_{k=0}^{n}\left(\begin{array}{l}
n \\
k
\end{array}\right) d_{1}^{n-k}\left(d_{2}^{k}(a)\right)=\sum_{k=0}^{n}\left(\begin{array}{l}
n \\
k
\end{array}\right) d_{2}^{n-k}\left(d_{1}^{k}(a)\right)
$$

for all $n$. In particular, when $n=2$, the above equation reduces to

$$
d_{1}^{2}(a)+2 d_{1}\left(d_{2}(a)\right)+d_{2}^{2}(a)=d_{2}^{2}(a)+2 d_{2}\left(d_{1}(a)\right)+d_{1}^{2}(a) .
$$

Thus we have $2 d_{2}\left(d_{1}(a)\right)=2 d_{1}\left(d_{2}(a)\right)$, and since $A$ has no 2-torsion, we obtain that $d_{2}\left(d_{1}(a)\right)=d_{1}\left(d_{2}(a)\right)$ for all $a \in A$. Hence $d_{1}$ and $d_{2}$ commute. From Lemma 3.4 it follows that $\sigma_{d_{1}+d_{2}}=\sigma_{d_{1}} \circ \sigma_{d_{2}}$, and now from Theorem 3.6, we obtain that $d_{1}+$ $d_{2} \in \Psi(G)$. Let $d \in \Psi(G)$; then $\sigma_{d} \in G$ and from Theorem 3.5 we know that $\sigma_{-d}$ is the inverse of $\sigma_{d}$. Then $\sigma_{-d} \in G$, and thus $-d=\Psi\left(\sigma_{-d}\right) \in \Psi(G)$. Hence $\Psi(G)$ is a subgroup of Der $A$ consisting of commuting derivations. Since $\Phi$ and $\Psi$ are inverses, it follows that $\left.\Psi\right|_{G}$ is a group isomorphism.

Remark 4.3. We recall from [4] that for a $\operatorname{ring} A$ with $\mathbb{Q} \subseteq A$, a Seidenberg automorphism over $A$ is an automorphism $E$ of $A[[T]]$ leaving $T$ fixed and reducing to the identity modulo $T$. Such an $E$ restricted to $A$ gives a derivation on $A$, and conversely every derivation on $A$ extends uniquely to a Seidenberg automorphism over $A$. Further, if $\mathbb{Q} \subseteq A$ then as noted in equation (2), $\psi_{A}: A[[T]] \rightarrow H A$ is an isomorphism and that if $E$ is a Seidenberg automorphism over $A$ and $d$ is the derivation on $A$ from 
$E$, then the diagram

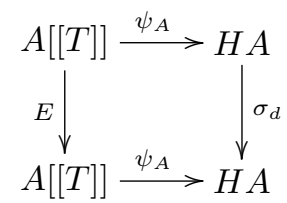

commutes. Thus a Hurwitz automorphism is a generalization of a Seidenberg automorphism to include the case when $\mathbb{Q} \nsubseteq A$, such as when the characteristic of $A$ is positive.

\section{Acknowledgements}

The authors thank Colas Bardavid, Alexey Ovchinnikov and Camilo Sanabria for their helpful remarks. The authors also thank the participants of the Kolchin Seminar in Differential Algebra for their constant support and encouragement throughout this project.

\section{References}

[1] W.F. Keigher, On the ring of Hurwitz series, Comm. Algebra 25 (1997), no. 6, 1845-1859.

[2] W.F. Keigher and F.L. Pritchard, Hurwitz series as formal functions, J. Pure Appl. Algebra 146 (2000), no. 3, 291-304.

[3] A. Seidenberg, Derivations and integral closure, Pacific J. Math. 16 (1966), no. $1,167-173$.

[4] W. Waterhouse, Notes toward higher differential algebra, J. Pure Appl. Algebra 7 (1976), no. 2, 121-132.

William F. Keigher keigher@rutgers.edu

Department of Mathematics and Computer Science, Rutgers University, 101 Warren Street, Newark, NJ 07102

Varadharaj R. Srinivasan v.ravisrinivasan@gmail.com

Department of Mathematical Sciences, Indian Institute of Science Education and Research, Mohali, Knowledge City, Sector 81, SAS Nagar, Punjab 140306, India 